PROCEEDINGS OF THE AMERICAN MATHEMATICAL SOCIETY

Volume 126, Number 5, May 1998, Pages 1479-1485

S 0002-9939(98)04198-7

\title{
CHAIN RULES FOR CODERIVATIVES OF MULTIVALUED MAPPINGS IN BANACH SPACES
}

\author{
A. JOURANI AND L. THIBAULT \\ (Communicated by Dale Alspach)
}

\begin{abstract}
Our basic object in this paper is to establish calculus rules for coderivatives of multivalued mappings between Banach spaces. We consider the coderivative which is associated to some geometrical approximate subdifferential for functions.
\end{abstract}

\section{INTRODUCTION}

In his paper [12] B.S. Mordukhovich developed several calculus rules for coderivatives of sum and composition of multivalued mappings (and also other operations). He has given many applications in optimization and control problems where coderivatives of multivalued mappings have to be considered in a natural way, and he has related the concept of coderivatives to the study of generalized equations. All the results are proved for finite dimensional spaces and are strongly based on an extremal principle for local extremal points of a system of two sets. This extremal principle was introduced and used by the author in [10] to prove calculus rules for subdifferentials of functions.

The aim of this paper is to establish calculus rules for coderivatives of multivalued mappings between general Banach spaces. We consider the coderivatives which are associated to some geometric approximate subdifferential for functions. This concept of geometric approximate subdifferential has been discovered by Ioffe [2][3], who showed that it is a good extension to the infinite dimensional setting of the Mordukhovich subdifferential. Our method is quite simple and is completely different from that of [12].

\section{Preliminaries}

Throughout the paper $X, Y$ and $Z$ will be Banach spaces, $X^{*}, Y^{*}$ and $Z^{*}$ their topological duals equipped with the weak-star topology $w^{*}$. We will denote by $B_{X}$ the closed unit ball of $X$ and by $d(\cdot, S)$ the distance function to a subset $S$ of $X$,

$$
d(x, S)=\inf _{u \in S}\|x-u\| .
$$

Received by the editors July 7, 1995 and, in revised form, October 30, 1996.

1991 Mathematics Subject Classification. Primary 49J52, 58C20.

Key words and phrases. Coderivatives, approximate subdifferentials, coderivatively compactly multivalued mappings. 
We will write $x \stackrel{f}{\rightarrow} x_{0}$ and $x \stackrel{S}{\rightarrow} x_{0}$ to express $x \rightarrow x_{0}$ with $f(x) \rightarrow f\left(x_{0}\right)$ and $x \rightarrow x_{0}$ with $x \in S$, respectively, and we will denote by $G r F$ the graph of a multivalued mapping $F: X \rightrightarrows Y$, i.e.,

$$
G r F=\{(x, y): y \in F(x)\} .
$$

If not otherwise specified, the norm in a product of two Banach spaces is defined by $\|(a, b)\|=\|a\|+\|b\|$.

We will use the notations in Ioffe [4]-[5].

Let $f: X \rightarrow \mathbb{R} \cup\{+\infty\}$ be a lower semicontinuous function in a neighbourhood of $x_{0} \in X$ with $f\left(x_{0}\right)<\infty$. The approximate subdifferential (see Ioffe [4]-[5]), which is an extension to the context of Banach spaces of the concept introduced by Mordukhovich [10]-[12] for finite dimensional spaces, is defined by

$$
\partial_{A} f\left(x_{0}\right)=\bigcap_{L \in \mathcal{F}(X)} \limsup _{\substack{f \rightarrow \\ \rightarrow} x_{o}} \partial^{-} f_{x+L}(x),
$$

where

$$
\begin{gathered}
\partial^{-} f(x)=\left\{x^{*} \in X^{*}:\left\langle x^{*}, h\right\rangle \leq d^{-} f(x ; h), \forall h \in X\right\}, \\
d^{-} f(x ; h)=\liminf _{\substack{u \rightarrow h \\
t \downarrow 0}} t^{-1}(f(x+t u)-f(x)) .
\end{gathered}
$$

Here, for $S \subset X, f_{S}$ denotes the function defined by

$$
f_{S}(x)= \begin{cases}f(x) & \text { if } \quad x \in S \\ +\infty & \text { otherwise }\end{cases}
$$

$\mathcal{F}(X)$ is the family of all finite dimensional subspaces of $X$, and

$$
\underset{\substack{f \\ x \rightarrow x_{o}}}{\limsup } \partial^{-} f_{x+L}(x)=\left\{x^{*} \in X^{*}: x^{*}=w^{*}-\lim x_{i}^{*}, x_{i}^{*} \in \partial f_{x_{i}+L}\left(x_{i}\right), \quad x_{i} \stackrel{f}{\rightarrow} x_{0}\right\},
$$

that is, the set of $w^{*}$-limits of all such nets.

The approximate coderivative of a multivalued mapping $F: X \rightrightarrows Y$ at a point $\left(x_{0}, y_{0}\right)$ of its graph $G r F$ is the multivalued mapping $D^{*} F\left(x_{0}, y_{0}\right): Y^{*} \rightrightarrows X^{*}$ defined by

$$
D^{*} F\left(x_{0}, y_{0}\right)\left(y^{*}\right)=\left\{x^{*}:\left(x^{*},-y^{*}\right) \in \mathbb{R}_{+} \partial_{A} d\left(x_{0}, y_{0} ; G r F\right)\right\} .
$$

For the distance function and for

$$
\partial_{\varepsilon}^{-} f(x)=\left\{x^{*} \in X^{*}:\left\langle x^{*}, h\right\rangle \leq d^{-} f(x ; h)+\varepsilon\|h\| \quad \forall h\right\},
$$

Ioffe [5] has given the following result.

Lemma 2.1. Let $C$ be a closed subset of $X$ containing $x_{0}$. Then

$$
\partial_{A} d\left(x_{0}, C\right)=\bigcap_{L \in \mathcal{F}(X)} \limsup _{\substack { C \\
\begin{subarray}{c}{C \\
\varepsilon \downarrow 0{ C \\
\begin{subarray} { c } { C \\
\varepsilon \downarrow 0 } }\end{subarray}} \partial_{\varepsilon}^{-} d_{x+L}(x, C) \cap(1+\varepsilon) B_{X^{*}} .
$$

Before stating Proposition 2.2, which will be one of the main tools of section 3, let us recall the following notion by Borwein and Strojwas [1]. A set $S \subset X$ is said to be compactly epi-Lipschitzian at $x_{0} \in S$ if there exist $\gamma>0$ and a \|\|$-$ compact set $H \subset X$ such that

$$
\left.S \cap\left(x_{0}+\gamma B_{X}\right)+t \gamma B_{X} \subset S-t H, \quad \text { for all } t \in\right] 0, \gamma[.
$$


We recall also that, following Jourani and Thibault [9], a multivalued mapping $F: X \rightrightarrows Y$ is partially coderivatively compact at $\left(x_{0}, y_{0}\right) \in G r F$ if the following condition is satisfied:

$$
\left\{\begin{array}{l}
\text { if there exist nets }\left(x_{i}, y_{i}\right) \stackrel{\operatorname{GrF}}{\rightarrow}\left(x_{0}, y_{0}\right) \text { and } \\
\left(x_{i}^{*}, y_{i}^{*}\right) \in \mathbb{R}_{+} \partial_{A} d\left(x_{i}, y_{i}, G r F\right) \text { such that }\left\|x_{i}^{*}\right\| \rightarrow 0 \text { and } y_{i}^{*} \rightarrow 0, \\
\text { then }\left\|y_{i}^{*}\right\| \rightarrow 0 .
\end{array}\right.
$$

Proposition 2.2 ([7]-[9]). Let $C$ and $D$ be two closed subsets of $X$ with $x_{0} \in$ $C \cap D$. Consider the following assertions:

(a) $D$ is compactly epi-Lipschitzian at $x_{0}$ and $\partial_{A} d\left(x_{0}, C\right) \cap\left(-\partial_{A} d\left(x_{0}, D\right)\right)=\{0\}$;

(b) the multivalued mapping $F: X \rightrightarrows X$ defined by

$$
F(x)= \begin{cases}-x+D & \text { if } x \in C, \\ \emptyset & \text { otherwise },\end{cases}
$$

is partially coderivatively compact at $\left(x_{0}, 0\right)$, and ker $D^{*} F\left(x_{0}, 0\right)=\{0\}$;

(c) there exist $a>0$ and $r>0$ such that

$$
d(x, C \cap D) \leq a[d(x, C)+d(x, D)]
$$

for all $x \in x_{0}+r B_{X}$;

(d) there exists $b>0$ such that

$$
\partial_{A} d\left(x_{0}, C \cap D\right) \subset b\left[\partial_{A} d\left(x_{0}, C\right)+\partial_{A} d\left(x_{0}, D\right)\right] .
$$

Then $(a) \Longrightarrow(b) \Longrightarrow(c) \Longrightarrow(d)$.

\section{Chain Rules}

In this section we are going to prove a general chain rule for multivalued mappings, and we will show how other formulas may be derived from it.

Theorem 3.1. Let $G: X \rightrightarrows Y$ and $F: Y \rightrightarrows Z$ be two multivalued mappings, and $\left(x_{0}, z_{0}\right) \in G r F \circ G$. Suppose that the following assumptions are fulfilled:

i) For each $y_{0} \in G\left(x_{0}\right) \cap F^{-1}\left(z_{0}\right)$ there are $a>0$ and $r>0$ such that

$$
d(x, y, z, G r G \times Z \cap X \times G r F) \leq a[d(x, y, G r G)+d(y, z, G r F)]
$$

for all $(x, y, z) \in\left(x_{0}, y_{0}, z_{0}\right)+r B_{X \times Y \times Z}$.

ii) For any net $\left(\left(x_{i}, z_{i}\right)\right)$ of $G r(F \circ G)$ converging to $\left(x_{0}, z_{0}\right)$ there exist some net (yi $y_{i}$ with $y_{i} \in G\left(x_{i}\right) \cap F^{-1}\left(z_{i}\right)$ and some point $y_{0} \in G\left(x_{0}\right) \cap F^{-1}\left(z_{0}\right)$ which is a cluster point of $\left(y_{i}\right)$.

Then for all $z^{*} \in Z^{*}$

$$
D^{*}(F \circ G)\left(x_{0}, z_{0}\right)\left(z^{*}\right) \subset \bigcup_{y_{0} \in G\left(x_{0}\right) \cap F^{-1}\left(z_{0}\right)} D^{*} G\left(x_{0}, y_{0}\right) \circ D^{*} F\left(y_{0}, z_{0}\right)\left(z^{*}\right) .
$$

Proof. Set $C=G r G \times Z$ and $D=X \times G r F$. We start by showing that

$$
\left\{\begin{array}{l}
\text { if }\left(x^{*}, z^{*}\right) \in \partial_{A} d\left(x_{0}, z_{0}, G r(F \circ G)\right) \text { then there exists } y_{0} \in G\left(x_{0}\right) \cap F^{-1}\left(z_{0}\right) \\
\text { such that }\left(x^{*}, 0, z^{*}\right) \in \partial_{A} d\left(x_{0}, y_{0}, z_{0}, C \cap D\right) .
\end{array}\right.
$$

Fix any $\left(x^{*}, z^{*}\right) \in \partial_{A} d\left(x_{0}, z_{0}, G r(F \circ G)\right)$, and put $S=c l(G r(F \circ G))$. Then for each $L=L^{1} \times L^{2} \in \mathcal{F}(X) \times \mathcal{F}(Z)$, by Lemma 2.1 , there are nets $\left(u_{i, L}, v_{i, L}\right)_{i} \stackrel{S}{\longrightarrow}\left(x_{0}, z_{0}\right)$, 
$\left(\varepsilon_{i, L}\right)_{i} \longrightarrow 0^{+}$with $2 \varepsilon_{i, L}<1,\left(x_{i, L}^{*}\right)_{i} \stackrel{w^{*}}{\longrightarrow} x^{*}$ and $\left(z_{i, L}^{*}\right)_{i} \stackrel{w^{*}}{\longrightarrow} z^{*}$ such that for $j:=$ $(i, L)$ in $J:=I \times \mathcal{F}(X) \times \mathcal{F}(Z)$

$$
\left(x_{j}^{*}, z_{j}^{*}\right) \in \partial_{\varepsilon_{j}}^{-} f_{\left(u_{j}, v_{j}\right)+L}\left(u_{j}, v_{j}\right) \text { and }\left\|\left(x_{j}^{*}, z_{j}^{*}\right)\right\| \leq 1+\varepsilon_{j},
$$

where $f(x, z)=d(x, z, S)$. So from Lemma 1 in [3] and Proposition 2.4.3 in [2], we get some $\left.r_{j} \in\right] 0, \frac{1}{3} \varepsilon_{j}$ [ such that the function

$$
\begin{aligned}
(x, z) \longmapsto f(x, z)- & \left\langle x_{j}^{*}, x-u_{j}\right\rangle-\left\langle z_{j}^{*}, z-v_{j}\right\rangle \\
& +2 \varepsilon_{j}\left(\left\|x-u_{j}\right\|+\left\|z-v_{j}\right\|\right)+3 d\left(x-u_{j}, z-v_{j}, L\right)
\end{aligned}
$$

attains a minimum at $\left(u_{j}, v_{j}\right)$ over $\left(u_{j}, v_{j}\right)+3 r_{j} B_{X \times Z}$. Take $\left(x_{j}^{\prime}, z_{j}^{\prime}\right) \in G r(F \circ G)$ with $\left\|\left(x_{j}^{\prime}, z_{j}^{\prime}\right)-\left(u_{j}, v_{j}\right)\right\| \leq r_{j}^{2}$. Choose by condition ii) some isotone mapping $k \longmapsto \alpha(k)=\left(i_{k}, L_{k}\right)$ from some directd set $K$ into $J$ (directed in the standard way) and some net $y_{j}^{\prime} \in G\left(x_{j}^{\prime}\right) \cap F^{-1}\left(z_{j}^{\prime}\right)$ with $\left(y_{\alpha(k)}^{\prime}\right)$ converging to some $y_{0} \in$ $G\left(x_{0}\right) \cap F^{-1}\left(z_{0}\right)$. Putting

$$
\begin{aligned}
\varphi_{j}(x, y, z)=d(x, y, z, C \cap D) & -\left\langle x_{j}^{*}, x-x_{j}^{\prime}\right\rangle-\left\langle z_{j}^{*}, z-z_{j}^{\prime}\right\rangle \\
& +2 \varepsilon_{j}\left(\left\|x-x_{j}^{\prime}\right\|+\left\|z-z_{j}^{\prime}\right\|\right)+3 d\left(x-x_{j}^{\prime}, z-z_{j}^{\prime}, L\right)
\end{aligned}
$$

for $j=(i, L)$ and using (3.2), one obtains $\varphi_{j}\left(x_{j}^{\prime}, y_{j}^{\prime}, z_{j}^{\prime}\right) \leq \varphi_{j}(x, y, z)+7 r_{j}^{2}$ for all $(x, y, z)$ in $\left(x_{j}^{\prime}, y_{j}^{\prime}, z_{j}^{\prime}\right)+2 r_{j} B$. By Ekeland's variational principle there exists $\left(x_{j}, y_{j}, z_{j}\right)$ in $\left(x_{j}^{\prime}, y_{j}^{\prime}, z_{j}^{\prime}\right)+r_{j} B$ such that for all $(x, y, z)$ in $\left(x_{j}^{\prime}, y_{j}^{\prime}, z_{j}^{\prime}\right)+2 r_{j} B$ one has

$$
\varphi_{j}\left(x_{j}, y_{j}, z_{j}\right) \leq \varphi_{j}(x, y, z)+7 r_{j}\left(\left\|x-x_{j}\right\|+\left\|y-y_{j}\right\|+\left\|z-z_{j}\right\|\right) .
$$

Therefore for $s_{k}:=2 \varepsilon_{\alpha(k)}+7 r_{\alpha(k)}$ one gets

$$
\left(x_{\alpha(k)}^{*}, 0, z_{\alpha(k)}^{*}\right) \in \partial_{A} d\left(x_{\alpha(k)}, y_{\alpha(k)}, z_{\alpha(k)}, C \cap D\right)+s_{k} B+\left(L_{k}^{1}\right)^{\perp} \times\{0\} \times\left(L_{k}^{2}\right)^{\perp},
$$

and hence $\left(x^{*}, 0, z^{*}\right) \in \partial_{A} d\left(x_{0}, y_{0}, z_{0}, C \cap D\right)$.

Consider now $z^{*} \in Z^{*}$ and $x^{*} \in D^{*}(F \circ G)\left(x_{0}, z_{0}\right)\left(z^{*}\right)$. Choose $r>0$ such that $r\left(x^{*},-z^{*}\right) \in \partial_{A} d\left(x_{0}, z_{0}, G r(F \circ G)\right)$. By (3.1) there exists $y_{0} \in G\left(x_{0}\right) \cap F^{-1}\left(z_{0}\right)$ such that $r\left(x^{*}, 0,-z^{*}\right) \in \partial d\left(x_{0}, y_{0}, z_{0}, C \cap D\right)$. So the result follows by applying Proposition 2.2 and the obvious equalities

$$
\partial_{A} d\left(x_{0}, y_{0}, z_{0}, C\right)=\partial_{A} d\left(x_{0}, y_{0}, G r G\right) \times\{0\}
$$

and

$$
\partial_{A} d\left(x_{0}, y_{0}, z_{0}, D\right)=\{0\} \times \partial_{A} d\left(y_{0}, z_{0}, G r F\right) .
$$

We consider an important corollary of Theorem 3.1 that gives more verifiable conditions.

Corollary 3.2. Let $G: X \rightrightarrows Y$ and $F: Y \rightrightarrows Z$ be two multivalued mappings with closed graphs. Suppose that, in addition to ii) in Theorem 3.1, the following assumptions are fulfilled:

$\left.i^{\prime}\right)$ For each $y_{0} \in G\left(x_{0}\right) \cap F^{-1}\left(z_{0}\right)$ the set $G r F$ (or $G r G$ ) is compactly epiLipschitzian at $\left(y_{0}, z_{0}\right)$ (resp. $\left.\left(x_{0}, y_{0}\right)\right)$.

iii) For all $y_{0} \in G\left(x_{0}\right) \cap F^{-1}\left(z_{0}\right)$

$$
\left[y^{*} \in D^{*} F\left(y_{0}, z_{0}\right)(0) \text { and } 0 \in D^{*} G\left(x_{0}, y_{0}\right)\left(y^{*}\right)\right] \Longrightarrow y^{*}=0 .
$$

Then the conclusion of Theorem 3.1 is valid. 
Proof. Set $C=G r G \times Z$ and $D=X \times G r F$ and consider the multivalued mapping $M: X \times Y \times Z \rightrightarrows X \times Y \times Z$ defined by

$$
M(x, y, z)= \begin{cases}-(x, y, z)+D & \text { if }(x, y, z) \in C, \\ \emptyset & \text { otherwise }\end{cases}
$$

The proof is concluded by applying Proposition 2.2 and Theorem 3.1.

Another consequence of this theorem is the following result concerning the convex case.

Corollary 3.3. Let $G: X \rightrightarrows Y$ and $F: Y \rightrightarrows Z$ be two multivalued mappings with closed and convex graphs. Suppose that condition ii) of Theorem 3.1 is satisfied and that

$$
0 \in \operatorname{int}(G r G \times Z-X \times G r F) .
$$

Then the conclusion of Theorem 3.1 is valid.

Proof . Consider the multivalued mapping $M: X \times Y \times Z \rightrightarrows X \times Y \times Z$ defined by

$$
M(x, y, z)=\left\{\begin{array}{l}
-(x, y, z)+X \times G r F \quad \text { if }(x, y, z) \in G r G \times Z, \\
\emptyset \quad \text { otherwise }
\end{array}\right.
$$

Then $G r M$ is closed and convex, and $0 \in \operatorname{int} \Phi(X \times Y \times Z)$. So, by Proposition 4.2 in [9], $M$ is partially coderivatively compact at all points $\left(x_{0}, y_{0}, z_{0}, 0\right)$ satisfying $y_{0} \in G\left(x_{0}\right) \cap F^{-1}\left(z_{0}\right)$, and the proof can be completed by applying Proposition 2.2 and Theorem 3.1.

Now we use Theorem 3.1 to obtain calculus rules for the sum of two multivalued mappings.

Theorem 3.4. Let $F_{1}, F_{2}: X \rightrightarrows Y$ be two multivalued mappings with closed graphs, and let $z_{0} \in F_{1}\left(x_{0}\right)+F_{2}\left(x_{0}\right)$. Suppose that the following conditions are satisfied:

$\left.i^{\prime}\right) G r F_{1}$ is compactly epi-Lipschitzian at all points $\left(x_{0}, z_{0}-y_{0}\right) \in G r F_{1}$ with $y_{0} \in F_{2}\left(x_{0}\right)$.

$\left.i i^{\prime}\right)$ For any net $\left(x_{i}, z_{i}\right) \stackrel{\operatorname{Gr}\left(F_{1}+F_{2}\right)}{\longrightarrow}\left(x_{0}, z_{0}\right)$ there exist convergent subnets $\left(v_{\alpha(j)}\right)$ and $\left(w_{\alpha(j)}\right)$ such that $z_{\alpha(j)}=v_{\alpha(j)}+w_{\alpha(j)}, v_{\alpha(j)} \in F_{1}\left(x_{\alpha(j)}\right)$ and $w_{\alpha(j)} \in F_{2}\left(x_{\alpha(j)}\right)$.

iii) For all $y_{0} \in F_{2}\left(x_{0}\right)$ with $z_{0}-y_{0} \in F_{1}\left(x_{0}\right)$,

$$
D^{*} F_{1}\left(x_{0}, z_{0}-y_{0}\right)(0) \cap\left(-D^{*} F_{2}\left(x_{0}, y_{0}\right)(0)\right)=\{0\} .
$$

Then for $A\left(x_{0}, z_{0}\right)=\left\{y_{0} \in F_{2}\left(x_{0}\right): z_{0}-y_{0} \in F_{1}\left(x_{0}\right)\right\}$ we have for all $y^{*} \in Y^{*}$

$$
D^{*}\left(F_{1}+F_{2}\right)\left(x_{0}, z_{0}\right)\left(y^{*}\right) \subset \bigcup_{y_{0} \in A\left(x_{0}, z_{0}\right)} D^{*} F_{1}\left(x_{0}, z_{0}-y_{0}\right)\left(y^{*}\right)+D^{*} F_{2}\left(x_{0}, y_{0}\right)\left(y^{*}\right) .
$$

The proof of this theorem will be derived from Theorem 3.1 and the following lemma.

Lemma 3.5. Let $F_{1}, F_{2}: X \rightrightarrows Y$ be two multivalued mappings, and consider the multivalued mappings $F$ and $G$ defined by

$$
\left.F(x, y)=y+F_{1}(x), \quad G(x)=\{x\} \times F_{2}(x)\right) .
$$


Then for $y_{0} \in F_{2}\left(x_{0}\right)$ with $z_{0}-y_{0} \in F_{1}\left(x_{0}\right)$ we have

$\left(x^{*}, y^{*}, z^{*}\right) \in \partial_{A} d\left(x_{0}, y_{0}, z_{0}, G r F\right) \Longrightarrow y^{*}=-z^{*},\left(x^{*}, z^{*}\right) \in \partial_{A} d\left(x_{0}, z_{0}-y_{0}, G r F_{1}\right)$

and

$$
\left(x^{*}, u^{*}, z^{*}\right) \in \partial_{A} d\left(x_{0}, x_{0}, y_{0}, G r G\right) \Longrightarrow\left(x^{*}+u^{*}, z^{*}\right) \in 2 \partial_{A} d\left(x_{0}, y_{0}, G r F_{2}\right) .
$$

Proof. Using the fact that $(u, y, v+y) \in G r F$ whenever $(u, v) \in G r F_{1}$, it is not difficult to see that

$$
d(x, y, z, G r F) \leq d\left(x, z-y, G r F_{1}\right)
$$

for all $(x, y, z) \in X \times Y \times Y$. Then for each $(x, y, z) \in \operatorname{cl}(G r G), f:=d(., G r F)$ and $f_{1}:=d\left(., G r F_{1}\right)$ one has for any $(u, v, w) \in X \times Y \times Y$

$$
\left.d^{-} f(x, y, z) ; u, v, w\right) \leq d^{-} f_{1}(x, z-y ; u, w-v) .
$$

So (3.3) easily follows from this inequality and Lemma 2.1 .

As $d(x, x, z, G r G) \leq 2 d\left(x, z, G r F_{2}\right)$ for all $(x, z) \in X \times Y$ (as easily seen), one obtains (3.4) in a similar way.

Proof of Theorem 3.4. Let $F$ and $G$ be as in Lemma 3.5. Then $y \in\left(F_{1}+F_{2}\right)(x)$ iff $y \in(F \circ G)(x)$. We easily show that $F$ and $G$ satisfy $i), i i)$ and $i i i)$ of Theorem 3.1 with $Y$ replaced by $X \times Y$ and $Z$ by $Y$. So let

$$
y^{*} \in Y^{*} \text { and } x^{*} \in D^{*}\left(F_{1}+F_{2}\right)\left(x_{0}, z_{0}\right)\left(y^{*}\right) .
$$

Then, by Theorem 3.1, there exist

$$
\left(u_{0}, y_{0}\right) \in G\left(x_{0}\right) \cap F^{-1}\left(z_{0}\right) \quad \text { and } \quad\left(u^{*}, v^{*}\right) \in D^{*} F\left(x_{0}, y_{0}, z_{0}\right)\left(y^{*}\right)
$$

such that $x^{*} \in D^{*} G\left(x_{0}, u_{0}, y_{0}\right)\left(u^{*}, v^{*}\right)$. Thus there exist $r_{1}>0$ and $r_{2}>0$ such that

$$
r_{1}\left(u^{*}, v^{*},-y^{*}\right) \in \partial_{A} d\left(x_{0}, y_{0}, z_{0}, G r F\right)
$$

and

$$
r_{2}\left(x^{*},-u^{*},-v^{*}\right) \in \partial_{A} d\left(x_{0}, u_{0}, y_{0}, G r G\right) .
$$

By Lemma $3.5 r_{1}\left(u^{*},-y^{*}\right) \in \partial_{A} d\left(x_{0}, z_{0}-y_{0}, G r F_{1}\right), v^{*}=y^{*}$ and

$$
2^{-1} r_{2}\left(x^{*}-u^{*},-v^{*}\right) \in \partial_{A} d\left(x_{0}, y_{0}, G r F_{2}\right)
$$

(because $u_{0}=x_{0}$ ). So we obtain

$$
x^{*}-u^{*} \in D^{*} F_{2}\left(x_{0}, y_{0}\right)\left(y^{*}\right) \text { and } u^{*} \in D^{*} F_{1}\left(x_{0}, z_{0}-y_{0}\right)\left(y^{*}\right) .
$$

Note that these calculus rules can be used to obtain subdifferential calculus for sums and compositions of mappings. This allows us to deduce the results in Ioffe [3]-[5], Mordukhovich [10]-[12], Jourani and Thibault [8] and Jourani [6].

For other applications to calculus rules, the reader can easily use Theorem 3.1 to get estimates of the coderivative of a multivalued mapping given, for instance, by $f(x) F(x):=\{f(x) y: y \in F(x)\}$, where $f$ is a real-valued mapping defined on $X$ and $F$ is a multivalued mapping from $X$ into $Y$. Many other examples can be considered.

We give another important application in a simple setting. More general cases will be treated in a forthcoming paper. 
Corollary 3.6. Let $G: X \rightrightarrows Y$ be a multivalued mapping with closed graph and $g: Y \rightarrow \mathbb{R}^{p}$ a locally Lipschitzian mapping. Suppose that $G$ satisfies ii) of Theorem 3.1 for $F(y)=g(y)+\mathbb{R}_{+}^{p}$ and also that for any $y_{0} \in G\left(x_{0}\right) \cap g^{-1}\left(z_{0}-\mathbb{R}_{+}^{p}\right)$

$$
\mathbb{R}_{+}^{p} \cap k e r D^{*} G\left(x_{0}, y_{0}\right) \circ D^{*} g\left(y_{0}\right)=\{0\} .
$$

Then there are $a>0$ and $r>0$ such that

$$
d\left(x,(g \circ G)^{-1}\left(z-\mathbb{R}_{+}^{p}\right)\right) \leq a d\left(z, g \circ G(x)+\mathbb{R}_{+}^{p}\right)
$$

for all $x \in\left(x_{0}+r B_{X}\right)$ and $z \in z_{0}+r B_{\mathbb{R}^{p}}$, with $d\left(z, g \circ G(x)+\mathbb{R}_{+}^{p}\right) \leq r$. (Here for a multivalued mapping $M, M^{-1}(S)=\{u: S \cap M(u) \neq \emptyset\}$.)

Proof. It suffices to apply Corollary 3.2 and Corollary 5.4 in [7].

After we completed this work, we received the paper [13] by Mordukhovich and Shao, which treats, by other methods in the spirit of [11] and [12], chain rules for limiting Fréchet coderivatives of multivalued mappings in Asplund spaces.

\section{ACKNOWLEDGMENT}

We thank a referee for some valuable comments.

\section{REFERENCES}

[1] J. M. Borwein and H. M. Strojwas, Tangential approximations, Nonlinear Anal. TMA, 9 (1985), 1347-1366. MR 87i:90320

[2] F. H. Clarke, Optimization and nonsmooth analysis, Wiley-Interscience, New York, (1983). MR 85m: 49002

[3] A. D. Ioffe, Approximate subdifferentials and applications 1: The finite dimensional theory, Trans. Amer. Math. Soc., 281 (1984), 389-416. MR 84m:49029

[4] A. D. Ioffe, Approximate subdifferentials and applications 2 : Functions on locally convex spaces, Mathematika, 33 (1986), 111-128. MR 87k:49028

[5] A. D. Ioffe, Approximate subdifferentials and applications 3 : Metric theory, Mathematika, 36 (1989), 1-38. MR 90g:49012

[6] A. Jourani, Intersection formulae and the marginal function in Banach spaces, J. Math. Anal. Appl., 192 (1995), 867-891. MR 96d:49022

[7] A. Jourani and L. Thibault, Verifiable conditions for openness and regularity of multivalued mappings in Banach spaces, Trans. Amer. Math. Soc., 347 (1995), 1255-1268. MR 95h:49021

[8] A. Jourani and L. Thibault, Extensions of subdifferential calculus rules in Banach spaces, Canadian J. Math., 48 (1996), 834-848. MR 97d:49018

[9] A. Jourani and L. Thibault, Coderivative of multivalued mappings, locally compact cones and metric regularity, (preprint 1995), to appear in Nonlinear Anal. Th. Meth. Appl.

[10] B. S. Mordukhovich, Approximation methods in problems of optimization and control, Nauka, Moscow, 1988 (in Russian; English translation to appear from Wiley-Interscience). MR 89m: 49001

[11] B. S. Mordukhovich, On variational analysis of differential inclusions, Editors A. D. Ioffe, M. Marcus and S. Reich, Pitman Research Notes in Mathematical Series, (1990), 199-213. CMP 93:01

[12] B. S. Mordukhovich, Generalized differential calculus for nonsmooth and set-valued mappings, J. Math. Anal. Appl., 182 (1994), 250-288. MR 95i:49029

[13] B. S. Mordukhovich and Y. Shao, Nonconvex differential calculus for infinite dimensional multifunctions, Set-Valued Anal. 4 (1996), 205-236. CMP 97:02

Université de Bourgogne, Laboratoire “Analyse Appliquée et Optimisation" B.P. 400, 21011 - Dijon CÉdex, France

E-mail address: jourani@satie.u-bourgogne.fr

Université Montpellier II, Laboratoire D’Analyse Convexe, Case Courrier 051 , 34095-Montpellier Cédex 5, France 